\title{
Aphalangy-syndactyly-microcephaly syndrome
}

INSERM

\section{Source}

INSERM. (1999). Orphanet: an online rare disease and orphan drug data base. Aphalangysyndactyly-microcephaly syndrome. ORPHA:1113

Aphalangy-syndactyly-microcephaly is an extremely rare malformation syndrome

characterized by the association of partial distal aphalangia with syndactyly, duplication of metatarsal IV, microcephaly, and mild intellectual disability. 